\title{
Single-center Experience of Outcomes of Tracheostomy in Children with Congenital Heart Disease
}

\author{
Geetha Challapudi, MD, ${ }^{*}$ Girija Natarajan, MD, ${ }^{\dagger}$ and Sanjeev Aggarwal, MD* \\ Divisions of ${ }^{*}$ Cardiology and ${ }^{\dagger}$ Neonatology, Carman and Ann Adams Department of Pediatrics, Children's Hospital of \\ Michigan, Wayne State University School of Medicine, Detroit, Mich, USA
}

\section{A B S T R A C T}

Objective. A subset of children with repaired congenital heart disease (CHD) may require tracheostomy for ongoing ventilatory support. Data on outcomes of children with CHD and tracheostomy are scarce. Our objectives were to describe indications for tracheostomy and outcomes, including readmission data in this population.

Methods. This is a retrospective chart review of children ( $<18$ years old) with CHD who underwent tracheostomy at a single center over a 12 -year period. Exclusion criteria were prematurity with isolated patent ductus arteriosus ligation. Outcomes until discharge and data on all readmissions after the initial discharge were reviewed.

Results. A total of 21 subjects with CHD underwent tracheostomy at a median (range) age of 4 (1-84) months and mean (standard deviation) weight of $7.2(5.9) \mathrm{kg}$. The most common indication for tracheostomy was tracheomalacia with ventilator-dependent respiratory failure (14/21 subjects), followed by subglottic stenosis (5) and vocal cord palsy (2). Genetic syndromes were present in 13 (62\%) subjects. The mean (standard deviation) post-tracheostomy length of stay was 55 (35) days. All subjects survived to discharge; 17 (81\%) required home ventilation. A total of 11 (52\%) subjects died during follow-up, all of whom were mechanically ventilated while three (14\%) children underwent successful decannulation. The mean number of nonelective readmissions decreased from 2.4/patient-year in the first year to 1.4/patient-year in the second year, respectively. The commonest reasons for readmission were respiratory deterioration, infections, and mechanical tracheostomy-related problems.

Conclusions. The majority of children with CHD who underwent tracheostomy did so for ventilator dependence and tracheomalacia and had coexisting genetic syndromes. About half the cohort died; among survivors, readmissions were common but decreased after the first year. These results underscore the ongoing mortality and morbidity risks faced by this vulnerable population.

Key Words. Congenital Heart Defects; Tracheostomy; Children

\section{Introduction}

T mprovements in surgical and cardiopulmonary 1 bypass techniques and postoperative care of children with congenital heart diseases (CHD) over the past few decades have resulted in improved survival. With increasing survival in this population, there is a small but growing proportion of children who require prolonged mechanical ventilation.

In a study of 172 young ( $\leq 3$ months) infants, prolonged ( $\geq 72$ hours) mechanical ventilation after cardiac surgery was required in 61 cases. $^{1}$ Risk adjustment for congenital heart surgery, nosocomial pneumonia, low cardiac output, postoperative cumulative positive fluid balance, and

Institution where the work was done: Children's Hospital of Michigan, Detroit, MI

Congenit Heart Dis. 2013;8:556-560 extubation failure were found to be independent risk factors for prolonged mechanical ventilation. ${ }^{1}$ Failed extubation, defined as reintubation within 48 hours of extubation, has been noted in $4.1 \%$ of pediatric intensive care unit patients, with children with CHD who failed extubation having the longest hospital stay. ${ }^{2}$ In another study, failed extubation within 24 hours was noted in $10 \%$ of children $\leq 36$ months following congenital heart surgery. ${ }^{3}$ Preoperative pulmonary hypertension, trisomy 21, and deep hypothermic cardiac arrest were independent risk factors for failed extubation. ${ }^{3}$ Tracheostomy and home ventilation are therapeutic options for children who fail extubation or require more prolonged ventilation. ${ }^{4}$ The limited published reports suggest that the incidence of tracheostomy and home ventilation in children with CHD is relatively low. ${ }^{5,6}$ In one 
center, 35 children with surgically corrected or palliated CHD were identified over a 16-year period, and in another, the reported rate of tracheostomy over a 5-year period from 2002 to 2007 was $0.2 \%(4 / 1660)$ of cardiac surgeries. ${ }^{5,6}$ There is a paucity of data on the indications, in-hospital, and follow-up readmission outcomes of children with CHD who underwent tracheostomy. We report our single-center experience with this population.

\section{Methods}

We undertook a retrospective chart and electronic medical record review of children ( $\leq 18$ years old) with CHD requiring corrective or palliative surgical repair in whom a tracheostomy was performed, at Children's Hospital of Michigan between January 2000 and December 2011. Preterm infants with ligation of the patent ductus arteriosus alone were excluded. We identified eligible children using an electronic database and the operative records of the otorhinolaryngology (ENT) service. The Institutional Review Board of the Human Investigation Committee at Wayne State University approved the study and waiver of parental consent and patient assent.

Data collected included demographic characteristics, details of CHD and surgical repair procedure, underlying genetic/neurologic condition, and operative data such as cross-clamp and cardiopulmonary bypass durations. The need for extracorporeal membrane oxygenation and left ventricular assist device was also abstracted. Outcomes until discharge and following discharge were abstracted from in-hospital and outpatient clinic records at our center. Nonelective rehospitalizations at our center were identified using the electronic medical records, and clinical documentation was used to ascertain reason for admission and outcomes.

At our center, all children with CHD following surgical repair are cared for in the pediatric intensive care unit by a multidisciplinary team of surgeons, intensivists, and cardiologists. Every attempt is made to extubate infants preoperatively and to extubate early postoperatively. Weaning of the ventilator is based on blood gases and saturations appropriate for the specific cardiac defect and surgery, hemodynamic stability, and clinical decision making. For subjects who fail attempted extubation, at least three such attempts are made prior to the decision for tracheostomy. A detailed evaluation for failed extubation including bron- choscopy, neuroimaging, echocardiogram, cardiac catheterization, and fluoroscopic evaluation of diaphragmatic movement is performed as indicated.

Statistical analysis used descriptive statistics (SPSS version 17.0, SPSS Inc., Chicago, IL, USA) including mean, standard deviation (SD), median and range, number and percent, as appropriate.

\section{Results}

A total of 21 patients over a 12 -year period were included in the study. Table 1 shows the baseline characteristics of the patient population. Cardiac surgery was performed at a median (range) age of 3 weeks ( 1 week-7 years); the mean (SD) cardiopulmonary bypass time was 179 (50) minutes and the cross-clamp time was 114 (65) minutes. Only one subject underwent CHD surgical repair after tracheostomy. Six subjects required multiple CHD surgical procedures prior to the tracheostomy. The repeat surgeries included an initial coarctation repair and pulmonary artery (PA) band followed by Damus-Kaye-Stansel procedure, PA angioplasty, and removal of PA band in a child with double outlet right ventricle, coarctation of aorta, and L-transposition of great arteries; Blalock-Taussig shunt followed by right ventricle to PA conduit, patch closure of the ventricular septal defect, and right PA angioplasty in a case of complete atrioventricular septal defect type A with tetralogy of Fallot; hybrid procedure followed by

Table 1. Demographic and Clinical Data of the Study Cohort

\begin{tabular}{ll}
\hline Characteristic & $\mathrm{n}=21$ \\
$\mathrm{n}(\%)$
\end{tabular}

Fallot; VSD, ventricular septal defect. 


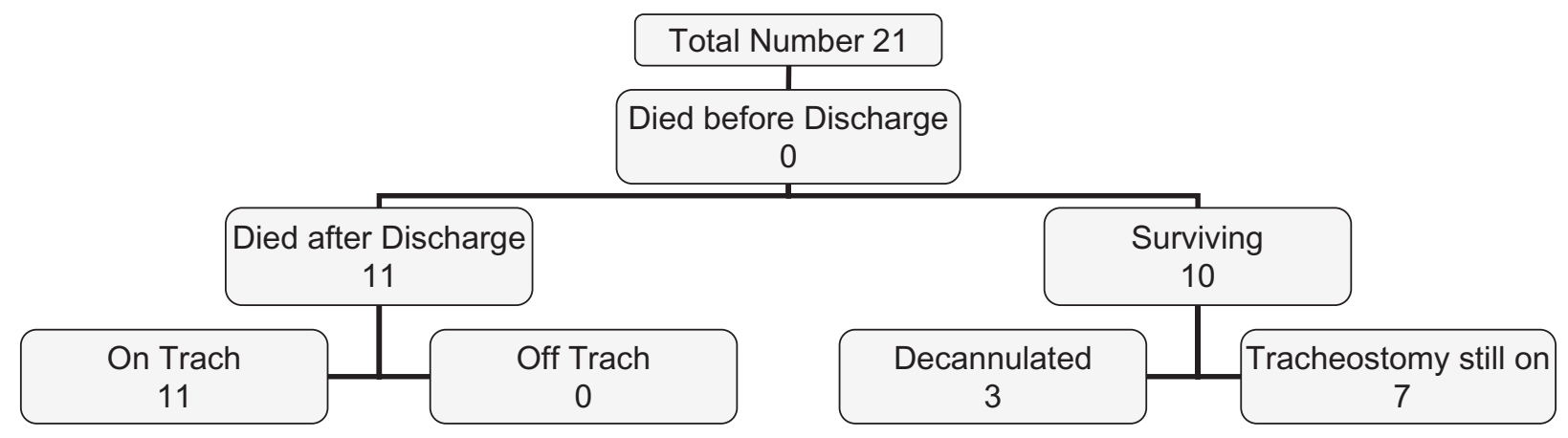

Figure 1. Flowchart of study cohort and associated outcomes.

heart transplantation for hypoplastic ascending aorta, interrupted aortic arch, ventricular septal defect, and pulmonary valve dysplasia; PA banding followed by heart transplantation and PA angioplasty in Truncus arteriosus type 1; BlalockTaussig shunt followed by patch tricuspid valve closure and reduction atrioplasty in Ebstein's anomaly and repair of complete atrioventricular septal defect followed by PA banding and mechanical common valve replacement and pacing lead placement. Following CHD repair, 3/21 $(14 \%)$ needed extracorporeal membrane oxygenation and 1/21 (4\%) needed left ventricular assist device. Mean (SD) duration of ventilation prior to tracheostomy was 39 (35) days. Synchronized intermittent mandatory ventilation was the commonest mode of ventilation in all except one case who also received high-frequency oscillation.

Direct laryngobronchoscopy was performed in all the patients; tracheomalacia with ventilatordependent respiratory failure was the most common (14/21) indication for tracheostomy, while subglottic stenosis (5) and vocal cord palsy (2) were diagnosed in a smaller numbers of subjects. Additional diagnostic modalities were cranial computed tomography and magnetic resonance imaging in one patient which showed a right parietal lobe infarct. The median (range) age at tracheostomy was 4 (1-84) months. The mean (SD) weights and heights at the time of the procedure were $7.2(5.9) \mathrm{kg}$ and $63.7(21.1) \mathrm{cm}$, respectively. Following tracheostomy, 12 ventilator-associated pneumonia episodes (eight Klebsiella pneumoniae, three Pseudomonas aeruginosa with three methicillin-resistant Staphylococcus aureus, one Acinetobacter baumanni) and two episodes of sepsis (coagulase-negative Staphylococcus, Enterobacter aerogenes) were diagnosed. Five subjects had arrhythmias (supraventricular tachycardia 3 , junctional ectopic tachycardia 1, and heart block 1), two had superior venacaval thrombosis, two had mediastinitis, and one had necrotizing enterocolitis. The mean (SD) length of hospital stay after the tracheostomy was 55 (35) days. Seventeen (81\%) subjects were discharged home on home ventilation and four on trach collar. Figure 1 is a flowchart describing outcomes of the study cohort. At the mean (SD) follow-up age of 2.5 (1.9) years, three patients were decannulated and 11 had died. Causes of death in 11 patients included sepsis (4), presumed cardiac arrest at home (2), respiratory failure due to pneumonia and tetralogy of Fallot with absent pulmonary valve (2), multiorgan failure (1), liver failure (1), and cardiac insufficiency in a case of unbalanced atrioventricular septal defect (1). Of those who died, two died during the first rehospitalization after tracheostomy, five died within 6 months, and four after 6 months of tracheostomy. Of the six infants who underwent multiple elective cardiac surgeries, four died at a median (range) age of 12.5 (7-23) months due to sepsis (3) or liver failure (1).

In the initial year following tracheostomy, 16 of the 21 subjects required nonelective rehospitalizations, with four needing more than five readmissions. The total number of nonelective rehospitalizations in the first year was 51 or 2.4 per patient year for a median (range) duration of 5 (1-43) days. Reasons for rehospitalizations in the first year following tracheostomy included respiratory deterioration in 27 (53\%), mechanical obstruction or dislodgement of the tracheostomy tube in $8(16 \%)$, bleed from the site in $2(4 \%)$, sepsis in $1(2 \%)$, and other infections in $13(25 \%)$ cases. In the second year, the 11 surviving subjects had 16 readmissions, or 1.4 per patient-year for a median (range) of 5 (1-11) days. Four of the 10 surviving subjects who had follow-up for more than 2 years had 20 late readmissions (beyond 2 years of the tracheostomy) for a median duration 
of 4 (1-33) days. Reinterventions for airway were frequently required: tracheal dilation in five cases, excision of granuloma in five cases, chemical cauterization in three cases, and laser therapy in three cases.

\section{Discussion}

The indications and outcomes of tracheostomy in children with CHD have been described in a few previous studies. In one study, four children, two of whom had single ventricle physiology, underwent tracheostomies over a 5 -year study period. ${ }^{6}$ Three had underlying genetic syndromes and one had a severe neurologic deficit after a cardiac arrest. Bronchomalacia and bilateral diaphragmatic palsy were additional findings in two children. The time to tracheostomy following surgical repair ranged from 17 to 100 days. ${ }^{6}$ LoTempio and colleagues described 15 children (incidence of $0.5 \%)$, two of whom had single ventricle physiology, who underwent tracheostomy following CHD surgical repair over a 6 -year period. ${ }^{7}$ Diaphragmatic paralysis was the commonest indication; vocal cord palsy, subglottic stenosis, a combination of these or a cerebrovascular infarct were other indications for tracheostomy. ${ }^{7}$ The mean duration between CHD surgery and tracheostomy was 32 days with a range of $0-72$ days. One patient died and six were decannulated. Cotts and colleagues described outcomes of 59 children under age 2 years who required tracheostomy after congenital heart surgery from a single center. ${ }^{8}$ Median time from surgery to tracheostomy was 36 days; genetic syndrome or noncardiac morbidities were present in $40 \%$ subjects. Intermediate term survival to a median duration of 25 months was $53 \% .{ }^{8}$ Hoskote and colleagues reviewed 37 children who underwent tracheostomy over a 4-year period, 24 of whom were following CHD surgery. ${ }^{9}$ Cardiac insufficiency, tracheobronchomalacia, diaphragmatic palsy, and new central nervous system events were the commonest reasons for prolonged ventilation. The interval between the primary surgery and tracheostomy was 27 (range 3-114) days. Twelve of the 24 children with CHD died; one patient had a local infection and decannulation was successful in 15/37 children. ${ }^{9}$ In another study involving 35 children with CHD surgery who underwent tracheostomy and home mechanical ventilation, $66 \%$ survived and 23\% were off the ventilator. ${ }^{5}$ Children with more complex CHD lesions, single ventricle physiology, or greater Risk Adjustment for Congenital Heart
Surgery scores had higher mortality and less weaning off ventilation. ${ }^{5}$ Our results are broadly concordant with these data; underlying genetic, airway, or rarely, neurologic issues were typical reasons for tracheostomy. The cardiac lesions varied widely and prolonged postoperative ventilation was the norm. Our mortality rate of about $50 \%$ was similar to the reports by Hoskote et al. and Cotts et al. and higher than the others, although our follow-up duration was longer. ${ }^{8,9} \mathrm{We}$ speculate that the higher mortality in our study was because of associated genetic syndromes in the majority of our subjects. Decannulation was successful in a small subset of our subjects, which suggests that some airway lesions and respiratory and cardiac insufficiency may improve over time with growth and cardiac recovery. In our dataset, there were no patients with single ventricle physiology.

Our finding of a large readmission burden in children with CHD following tracheostomy is novel. Hospital readmissions, especially in the first year following tracheostomy, were frequent and often unrelated to the CHD. In a previous study, among 70 children who underwent tracheostomies for varied indications at a single center, $63 \%$ were readmitted within 6 months, with $11 \%$ requiring four or more readmissions. ${ }^{10}$ Other studies on the pediatric home ventilation population have reported nonelective readmission rates of $40 \%$, with half occurring within the first 3 months after discharge. ${ }^{11}$ It has been previously reported that "technology-dependent" children with tracheostomies require considerable hospital resources use. $^{12}$

One limitation of our retrospective review was the absence of consistent clinical protocols to guide indications or timing of tracheostomy. Nonetheless, the current study adds to the slim body of literature on tracheostomy-related outcomes in children with repaired CHD. Tracheostomy is required in a small subset of patients with $\mathrm{CHD}$ requiring prolonged ventilation and with underlying genetic lesions. The prognosis for survival appears guarded and readmissions need to be anticipated.

\section{Conclusions}

The majority of our cohort of children with CHD who underwent tracheostomy had an underlying genetic syndrome. Ventilator-dependent respiratory failure was overwhelmingly the commonest reason for tracheostomy. While half the cohort 
died, a quarter of survivors were decannulated and many were off the ventilator at follow-up. Our data underscore that readmissions for respiratory deterioration, mechanical tracheostomy-related complications, or infections were frequent in the first 2 years and decreased progressively over the years. Further investigation into approaches to improve outcomes in a pediatric population with CHD is warranted. For example, the advantages of early tracheostomy (within 10 postoperative days) in reducing morbidity, length of stay, sternal wound infection, and in-hospital mortality have been demonstrated in adults with coronary artery bypass graft and/or valve surgery. ${ }^{13}$ Similarly, neurocritical adults with traumatic brain injury or stroke who underwent early (9 days or less) tracheostomy were found to have shorter duration of mechanical ventilation, ICU stay, and less pneumonia, than those with later tracheostomy. ${ }^{14}$ The optimal timing of tracheostomy in children with CHD who require prolonged ventilation is unclear.

\section{Authors' Contributions}

Geetha Challapudi, MD: acquisition and interpretation of the data.

Girija Natarajan, MD: data interpretation, drafting the paper, and revising it critically.

Sanjeev Aggarwal, MD: study design, analysis and interpretation of data and manuscript critical revision.

Corresponding Author: Sanjeev Aggarwal, MD, Division of Cardiology, Carman and Ann Adams Department of Pediatrics, Children's Hospital of Michigan, Wayne State University School of Medicine, 3901 Beaubien Boulevard, Detroit, MI 48201, USA. Tel: $313-$ 745-6105; Fax: 313-993-0894; E-mail: ssanjeev@ dmc.org

Conflict of interest: No conflict of interest, grants, or other financial support.

Accepted in final form: Fanuary 21, 2013.

\section{References}

1 Shi S, Zhao Z, Liu X, et al. Perioperative risk factors for prolonged mechanical ventilation following cardiac surgery in neonates and young infants. Chest. 2008;134:768-774.

2 Baisch SD, Wheeler WB, Kurachek SC, Cornfield DN. Extubation failure in pediatric intensive care incidence and outcomes. Pediatr Crit Care Med. 2005;6:312-318.

3 Harrison AM, Cox AC, Davis S, Piedmonte M, Drummond-Webb JJ, Mee RB. Failed extubation after cardiac surgery in young children: prevalence, pathogenesis, and risk factors. Pediatr Crit Care Med. 2002;3:148-152.

4 Edwards JD, Kun SS, Keens TG. Outcomes and causes of death in children on home mechanical ventilation via tracheostomy: an institutional and literature review. 7 Pediatr. 2010;157:955-959.e2.

5 Edwards JD, Kun SS, Keens TG, Khemani RG, Moromisato DY. Children with corrected or palliated congenital heart disease on home mechanical ventilation. Pediatr Pulmonol. 2010;45:645-649.

6 Rossi AF, Fishberger S, Hannan RL, et al. Frequency and indications for tracheostomy and gastrostomy after congenital heart surgery. Pediatr Cardiol. 2009;30:225-231.

7 LoTempio MM, Shapiro NL. Tracheotomy tube placement in children following cardiothoracic surgery: indications and outcomes. Am 7 Otolaryngol. 2002;23:337-340.

8 Cotts T, Hirsch J, Thorne M, Gajarski R. Tracheostomy after pediatric cardiac surgery: frequency, indications, and outcomes. 7 Thorac Cardiovasc Surg. 2011;141:413-418.

9 Hoskote A, Cohen G, Goldman A, Shekerdemian L. Tracheostomy in infants and children after cardiothoracic surgery: indications, associated risk factors, and timing. 7 Thorac Cardiovasc Surg. 2005; 130:1086-1093.

10 Graf JM, Montagnino BA, Hueckel R, McPherson ML. Pediatric tracheostomies: a recent experience from one academic center. Pediatr Crit Care Med. 2008;9:96-100.

11 Kun SS, Edwards JD, Ward SL, Keens TG. Hospital readmissions for newly discharged pediatric home mechanical ventilation patients. Pediatr Pulmonol. 2012;47:409-414.

12 Berry JG, Graham DA, Graham RJ, et al. Predictors of clinical outcomes and hospital resource use of children after tracheotomy. Pediatrics. 2009;124: 563-572.

13 Devarajan J, Vydyanathan A, Xu M, et al. Early tracheostomy is associated with improved outcomes in patients who require prolonged mechanical ventilation after cardiac surgery. 7 Am Coll Surg. 2012;214: 1008-1016.e4.

14 Gandia-Martinez F, Martinez-Gil I, Andaluz-Ojeda D, Bobillo de Lamo F, Parra-Morais L, DiezGutierrez F. [Analysis of early tracheostomy and its impact on development of pneumonia, use of resources and mortality in neurocritically ill patients]. Neurocirugia (Astur). 2010;21:211-221. 